\title{
Sampling and Reconstruction of Zero-Order Hold Signals by Parallel RC Filters"
}

\author{
Juuso T. Olkkonen ${ }^{1}$, Hannu Olkkonen² \\ ${ }^{1}$ VTT Technical Research Centre of Finland, Espoo, Finland; ${ }^{2}$ Department of Physics and Mathematics, University of Eastern \\ Finland, Kuopio, Finland. \\ Email: juuso.olkkonen@vtt.fi, hannu.olkkonen@uef.fi
}

Received April 1 ${ }^{\text {st }}, 2011$; Revised May 12 ${ }^{\text {th }}, 2011$; Accepted May 25 $5^{\text {th }}, 2011$.

\begin{abstract}
In this work we describe a reconstruction algorithm for zero-order hold ( $\mathrm{ZOH}$ ) waveforms measured by a parallel sampling scheme. In the method the $\mathrm{ZOH}$ signal is fed to a parallel network consisting of resistor-capacitor $(R C)$ filters, whose outputs are sampled simultaneously. The algorithm reconstructs $N$ previous samples of the input signal from output samples of $N$ parallel $R C$ circuits. The method is especially useful in sampling and reconstruction of the $\mathrm{ZOH}$ signals produced by the digital-to-analog converters. Using the parallel sampling method the sampling rate of the analog-to-digital converters can be increased by a factor of $N$. We discuss a variety of applications such as reconstruction of ZOH pulse sequences produced by ultra wide band (UWB) transmitters.
\end{abstract}

Keywords: Sampling, Interpolation, UWB, Analog-to-Digital Converters, Microprocessors, VLSI

\section{Introduction}

The present sampling methods are based on the Shannon's famous theorem [1], which concerns the sampling of the band-limited signals at equidistant time intervals. However, for example the measurement of ultra wide band (UWB) pulses gives rise to an extended demand for the signal sampling devices. The analog-to-digital converters (ADCs) have a limited conversion time, which is not sufficient for sampling of the short term transients. In signal processing society the sequential sampling scheme based on the finite rate of innovation (FRI) has been an object of active interest for reconstruction of the UWB pulses and other discontinuities [2-6]. In FRI methods the transient signal is fed to an analogue network, which broadens the signal for sampling with an ADC. The reconstruction algorithm is based on the ad hoc knowledge of the signal waveform. In our previous work [7] we introduced a new method for sampling and reconstruction of continuous transient waveforms. The signal is fed to the parallel network consisting resistor-capacitor (RC) filters. The outputs of the RC filters are sampled simultaneously. $N$ signal samples can be reconstructed from the single samples of $N$ parallel RC circuits. The reconstruction algorithm was based on the replacement of the This work was supported by the National Technology Agency of Finland (TEKES). convolution integral by the Riemann sum. Recently we observed that in reconstruction of transient pulse sequences, the Riemann sum is too rough estimate for the convolution integral. Especially when the signal is produced by the digital-to-analog converter (DAC), the reconstruction of the $\mathrm{ZOH}$ (piecewise constant) signal may involve unpredictable errors. In the following we present an algorithm for computation of the convolution integral. We describe the parallel RC network, develop the reconstruction algorithm and describe potential applications of the parallel sampling scheme.

\section{Parallel Sampling Scheme for $\mathbf{Z O H}$ Signals}

A causal continuous-time signal $x(t)$ under consideration is defined as

$$
x(t)=\left\{\begin{array}{c}
x(t) \text { for } t \geq 0 \\
0 \text { for } t<0
\end{array} .\right.
$$

The signal $x(t)$ is fed to the network consisting of $N$ parallel RC filters shown in Figure 1. The exponential impulse responses of the RC filters are for $t \geq 0$

$$
h_{i}(t)=C \alpha_{i} A_{i} e^{-\alpha_{i} t}, i=1,2,, N
$$

where $\alpha_{i}=1 /\left(R_{i} C_{i}\right)$ and $C$ is the scaling constant. For the RC filter outputs, which are sampled simultaneously 


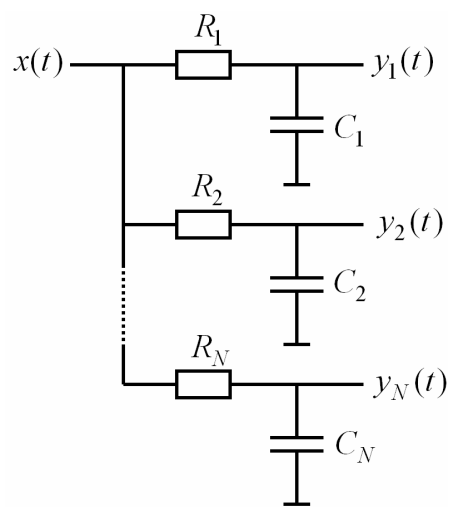

Figure 1. The s ampling scheme consisting of $N$ parallel RC filters.

at time $t_{0}$ the following is valid

$$
y_{i}\left(t_{0}\right)=x(t) * h_{i}(t)=\int_{0}^{t_{0}} x(\tau) h_{i}\left(t_{0}-\tau\right) \mathrm{d} \tau
$$

where * denotes convolution and $\tau$ the dummy integration variable. For computation of (3) the measurement instant $t_{0}$ is divided to $N$ equal intervals $\Delta t=t_{0} / N$. The discrete samples of the measurement signal are denoted by $x[n]=x(n \Delta t)$ and the outputs of the RC filters by $y_{i}[N]=y(N \Delta t)$. Supposing that the measurement signal is a ZOH signal yieded by DAC with a frequency $1 / \Delta t$ the convolution (3) can be integrated on $\Delta t$ intervals. We have

$$
\begin{aligned}
y_{i}[N] & =\int_{0}^{N \Delta t} x(\tau) C \alpha_{i} \exp \left[-\alpha_{i}(N \Delta t-\tau)\right] \mathrm{d} \tau \\
& =C \alpha_{i} \exp \left(-\alpha_{i} N \Delta t\right) \sum_{k=0}^{N-1} x[k] \int_{k \Delta t}^{(k+1) \Delta t} \exp \left(\alpha_{i} \tau\right) \mathrm{d} \tau \\
& =C\left[\exp \left(\alpha_{i} \Delta t\right)-1\right] \sum_{k=0}^{N-1} x[k] \exp \left[-\alpha_{i}(N-k) \Delta t\right]
\end{aligned}
$$

Using the short notation $u_{i}=\exp \left(-\alpha_{i} \Delta t\right)$ we obtain

$$
z_{i}[N]=\frac{1}{C\left[\exp \left(\alpha_{i} \Delta t\right)-1\right]} y_{i}[N]=\sum_{k=0}^{N-1} x[k] u_{i}^{N-k}
$$

which yields the following matrix/vector representation

$$
\left[\begin{array}{c}
z_{1}[N] \\
z_{2}[N] \\
\vdots \\
z_{N}[N]
\end{array}\right]=\left[\begin{array}{cccc}
u_{1}^{N} & u_{1}^{N-1} & \cdots & u_{1} \\
u_{2}^{N} & u_{2}^{N-1} & \cdots & u_{2} \\
\vdots & \vdots & \vdots & \vdots \\
u_{N}^{N} & u_{N}^{N-1} & \cdots & u_{N}
\end{array}\right]\left[\begin{array}{c}
x[0] \\
x[1] \\
\vdots \\
x[N-1]
\end{array}\right] \Leftrightarrow \mathbf{z}=\mathbf{u} \boldsymbol{x}
$$

Equation (6) includes a non-singular Vandermonde matrix having rank $N$. This enables us to solve the input signal $x[n], n=1,2, \cdots, N$, from the outputs $y_{i}[N]$ of $\mathrm{N}$ parallel RC filters, sampled at the time instant $t_{0}=N \Delta t$. The signal vector $\boldsymbol{x}$, containing $N$ discrete values, can be reconstructed from

$$
\boldsymbol{x}=\boldsymbol{u}^{-1} \mathbf{z}
$$

Since the inverse matrix $\boldsymbol{u}^{-1}$ depends only on the properties of RC filters and the discretization interval $\Delta t$, the signal reconstruction in later measurements is obtained by a single matrix-vector multiplication with the previously computed $\boldsymbol{u}^{-1}$.

The above formulation is relevant only for the reconstruction of the $N$ consecutive samples from the causal $\mathrm{ZOH}$ pulse sequence. For measurement of the next sequence of $N$ samples, the parallel RC network was modified by adding a FET switch and a sample and hold $(\mathrm{S} / \mathrm{H})$ circuit (Figure 2). The switch resets the output at the beginning of the measurement period at the time instant $t=0$ and the $\mathrm{S} / \mathrm{H}$ circuit samples the signal at $t_{0}=N \Delta t$. The next measurement period follows the same procedure. With this arrangement the $\mathrm{ZOH}$ waveform can be reconstructed without discontinuities.

The theoretical validation of the reconstruction algorithm $(6,7)$ was tested by simulating the parallel RC network (Figure 1) via the analog electronic circuit simulator (Spice). In the absence of noise the reconstruction algorithm recovered the $\mathrm{ZOH}$ waveforms with machine precision. When the random noise was added to the $\mathrm{ZOH}$ signal, the RC filters reduced clearly the noise level in the outputs. In the presence of noise the reconstruction produced $\mathrm{ZOH}$ signal levels, which matched the time averaged values

$$
x[k]=\frac{1}{\Delta t} \int_{k \Delta t}^{(k+1) \Delta t} x(t) \mathrm{d} t \quad k=0,1, \cdots, N-1
$$

\section{Experiments and Results}

The measurement equipment consisted of eight parallel RC-circuits equipped with FET switches and sample and hold $(\mathrm{S} / \mathrm{H})$ circuits. The $\alpha_{i} \Delta t$ parameter varied in the range $0.1, \cdots, 0.7$. The outputs of the $\mathrm{S} / \mathrm{Hs}$ were fed to the differential eight channel 12 bit ADC unit, hich had the $+/-5 \mathrm{~V}$ measurement range. Using this arrangement eight $\mathrm{ZOH}$ signal samples were reconstructed corresponding to the measurement interval $t \in[0, N \Delta t]$.

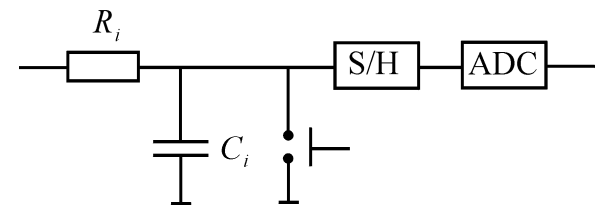

Figure 2. Parallel RC filt er equipped with the FET s witch and the sample and hold $(\mathrm{S} / \mathrm{H})$ circuit for sampling of $\mathrm{ZOH}$ waveforms. 
The piecewise constant test signals were produced by eight bit DAC, whose output was equipped with a unit gain buffer amplifier. The DAC pulse frequency varied between $10 \mathrm{kHz}$ and $1 \mathrm{MHz}$. All experimental tests were performed in a Faraday gage.

The $\mathrm{ZOH}$ pulse sequences comprised of sinusoidal, damped sinusoidal and various types of pulse waveforms. In all cases the algorithm reconstructed the $\mathrm{ZOH}$ signals with an average error being typically in the range 3.5 $4.2 \mathrm{mV}$. Most of the error was related to the quantization noise of the ADC unit.

\section{Discussion and Conclusions}

In our earlier work [7] we described a new measurement and sampling concept, which permits the reconstruction of short term transient signals. The key idea was the use of the parallel RC filters, whose outputs are measured simultaneously. The reconstruction algorithm was based on the replacement of the convolution integral (3) by the Riemann sum. In this work we reformulated the reconstruction algorithm for the $\mathrm{ZOH}$ waveforms. The key idea is that since the $\mathrm{ZOH}$ signal is convolved with the impulse response of the RC filter, the convolution integral can be calculated analytically integrating on $\Delta t$ intervals.

In our previous work we calibrated the measurements by denoting the impulse response of the RC circuit as

$$
h_{i}(t)=A_{i} e^{-\alpha_{i} t}, i=1,2, \cdots, N
$$

We wrote in [7] "In an ideal case, $A_{i}=\alpha_{i}$, but in practise they may slightly differ from each other”. To obtain relevant results each of the $A_{i}$ values were independently calibrated. The Riemann sum reconstruction method yielded the following result

$$
z_{i}[N]=\frac{1}{A_{i} \Delta t} y_{i}[N]
$$

It is interesting that for small $\alpha_{i} \Delta t$ values we may take two terms of the series expansion $\exp \left(\alpha_{i} \Delta t\right)=$ $1+\alpha_{i} \Delta t$ in (6). By equalizing $A_{i}=C \alpha_{i}$ we obtain

$$
\frac{1_{i}}{C\left[\exp \left(\alpha_{i} \Delta t\right)-1\right]} \Rightarrow \frac{1}{A_{i} \Delta t}
$$

i.e. series approximation reduces (5) to that yielded by Riemann sum (10). However, in most cases the series approximation is too rough for $\mathrm{ZOH}$ signals. The scaling constant $C$ affects the gain of the different channels and only one calibration coefficient is needed to calibrate all channels in parallel RC network.

In the present parallel sampling scheme the simultaneous measurements at the outputs of the RC filters are taken at $N \Delta t$ intervals. During that period the $\mathrm{ZOH}$ signal has $N$ transitions. The role of the RC network is to work as a low-pass filter by smoothing the abrupt changes of the pulse edges and filtering the random noise.

The reconstruction of the $N$ signal samples needs only one matrix-vector multiplication (7). The analytical formulae for the inversion of the Vandermonde matrix in (6) are well known [8-13]. The explicit solutions are preferred since they are more accurate than the general matrix inversion algorithms.

The conversion time of the ADCs is limited to the through output time of the comparator chain. In VLSI design the most effective configuration would be to use individual ADCs equipped with a S/H circuits to measure the parallel RC filters separately. By using $N$ parallel RC filter network the conversion time of each ADC can be prolonged to $N \Delta t$. This makes it possible to increase the sampling rate of the data acquisition system by a factor of $N$.

In test measurements we found only a tiny interference due to the operation of the FET switches. Obviously the RC filters effectively reduce most of the high frequency noise imposed on the signal.

The present method has plenty of applications including the measurement of pulse trains yielded by pulse lasers and ultra wide-band (UWB) pulse transmitters. Usually the UWB pulses are short transients and the information is coded to the appearance time of the pulses. The information may also be coded to the shape of the UWB pulses [14]. Using a single receiver several transmitters can be measured simultaneously e.g. in multisensor applications.

The present parallel sampling scheme can be seen as a special case of the setup called sparse or compressive sampling [15-20], which can be adapted to recover certain signals from far fewer measurements than conventional sampling methods require.

\section{Acknowledgements}

We are indebted to the anonymous referee for comments, which have improved the manuscript significantly.

\section{REFERENCES}

[1] M. Unser, “Sampling-50 Years after Shannon,” Proceedings of the IEEE, Vol. 88, No. 4, 2000, pp. 569-587. doi:10.1109/5.843002

[2] P. Marziliano, "Sampling Innovations," Ph.D. Dissertation, Ecole Polytechnique Federale de Lausanne, Switzerland, April 2001.

[3] M. Vetterli, P. Marziliano and T. Blu, "Sampling Signals with Finite Rate of Innovation," IEEE Transactions on Signal Process, Vol. 50, No. 6, 2002, pp. 1417-1428. doi:10.1109/TSP.2002.1003065 
[4] I. Maravic and M. Vetterli, "Sampling and Reconstruction of Signals with Finite Rate of Innovation in the Presence of Noise,” IEEE Transactions on Signal Process, Vol. 53, No. 8, 2005, pp. 2788-2805. doi:10.1109/TSP.2005.850321

[5] P. L. Dragotti, M. Vetterli and T. Blu, "Sampling Moments and Reconstructing Signals of Finite Rate of Innovation: Shannon Meets Strang-Fix," IEEE Transactions on Signal Process, Vol. 55, No. 5, 2007, pp. 1741-1757. doi:10.1109/TSP.2006.890907

[6] I. Maravic, J. Kusuma and M. Vetterli, "Low-Sapling Rate UWB Channel Characterization and Syncronization," Journal of Communications and Networks, Vol. 5, No. 4, 2003, pp. 319-327.

[7] H. Olkkonen and J. T. Olkkonen, "Measurement and Reconstruction of Transient Signals by Parallel Exponential Filters," IEEE Transactions on Circuits and Systems II, Vol. 57, No. 6, 2010, pp. 426-429. doi:10.1109/TCSII.2010.2048375

[8] J. T. Tou, "Determination of the Inverse Vander Monde Matrix,” IEEE Transactions on Automatic Control, Vol. 9, No. 3, 1964, p. 314. doi:10.1109/TAC.1964.1105716

[9] H. J. Wertz, "On the Numerical Inversion of a Recurrent Problem: The Vander Monde Matrix,” IEEE Transactions on Automatic Control, Vol. 10, No. 4, 1965, p. 492. doi:10.1109/TAC.1965.1098206

[10] S. H. Wu, "On the Inverse of Vander Monde Matrix," IEEE Transactions on Automatic Control, Vol. 11, No. 4, 1966, p. 769. doi:10.1109/TAC.1966.1098464

[11] V. E. Neagoe, "Inversion of the van der Monde Matrix," IEEE Signal Processing Letters, Vol. 3, No. 4, 1996, pp. 119-120. doi:10.1109/97.489066

[12] G. C. Reis, "A Matrix Formulation for the Inverse Vander Monde Matrix," IEEE Transactions on Automatic Con- trol, Vol. 12, No. 6, 1967, p. 793. doi:10.1109/TAC.1967.1098772

[13] I. Kaufman, "The Inversion of the VanderMonde Matrix and the Transformation to the Jordan Canonical Form," IEEE Transactions on Automatic Control, Vol. 14, No. 6, 1969, pp. 774-777. doi:10.1109/TAC.1969.1099332

[14] H. Olkkonen and J. T. Olkkonen, "Design of Orthogonal UWB Pulse Waveform for Wireless Multi-Sensor Applications," Wireless Sensor Network, Vol. 2, No. 11, 2010 , pp. 850-853. doi:10.4236/wsn.2010.211102

[15] J. R. Higgins, "Sampling Theory in Fourier and Signal Analysis: Foundations,” Clarendon Press, Oxford, 1996.

[16] L. Sbaiz, P. Vandewalle and M. Vetterli, “Groebner Basis Methods for Multichannel Sampling with Unknown Offsets," Applied and Computational Harmonic Analysis, Vol. 25, No. 3, 2008, pp. 277-294. doi:10.1016/j.acha.2007.11.003

[17] L. Baboulaz and P. L. Dragotti, "Distributed Acquisition and Image Super-Resolution Based on Continuous Moments from Samples," Proceedings of IEEE International Conference on Image Processing, Atlanta, 8-11 October 2006, pp. 3309-3312.

[18] H. T. Nguyen and M. N. Do, "Hybrid Filter Banks with Fractional Delays: Minimax Design and Application to Multichannel Sampling," IEEE Transactions on Signal Processing, Vol. 56, No. 7, 2008, pp. 3180-3190. doi:10.1109/TSP.2008.920489

[19] H. T. Nguyen and M. N. Do, "Robust Multichannel Sampling," Proceedings of IEEE International Conference on Image Processing, San Diego, 12-15 October 2008, pp. 653-656.

[20] E. J. Candes and M. B. Wakin, "An Introduction to Compressive Sampling,” IEEE Signal Processing, Vol. 25, No. 2, 2008, pp. 21-30. doi:10.1109/MSP.2007.914731 